\title{
Communications
}

\section{Note sur l'apiculture et les abeilles africanisées en Guyane française}

\author{
M. Canova ${ }^{1}$ \\ B. Gaucher ${ }^{1}$
}

CANOVA (M.), GAUCHER (B.). Note sur l'apiculture et les abeilles africantisées en Guyane française. Revue Élev. Méd. vét. Pays trop., 1991, 44 (4) : 497-500

L'apiculture est presque inexistante en Guyane française. Afin d'étudier les possibilités de développer cette activité, le comportement de l'abeille africanisée, les sources de pollen et de nectar et les niveaux de production ont été décrits en comparant trois milieux naturels différents : la mangrove, la savane et la forêt. Mots clés : Abeille Comportement Nlimentation Pollen - Nectar - Guyane française.

\section{Introduction}

Le plan de mise en valeur agricole de la Guyane décidé par le gouvernement français en 1975 consistait, après exploitation de la forêt pour l'industrie papetière, à créer des exploitations modernes en vue de satisfaire le marché local en productions fruitières et vivrières, ainsi qu'en viande. Dix années plus tard, les agriculteurs sont confrontés à de sérieux problèmes et la pérennité de leurs exploitations est menacée (coûts de production élevés, productivité faible, problèmes sanitaires).

L'apiculture, activité presque inexistante en Guyane française, pourrait-elle présenter une voie de diversification intéressante en assurant un revenu complémentaire décent?

Malgré l'invasion inéluctable des abeilles africanisées (Apis mellifera adansonii) depuis le Brésil $(6,10)$, ce pays frontalier est le premier Etat producteur sud américain de miel (30 000 t en 1988). Les rares introductions d'abeilles européennes (Apis mellifera ligustica) n'ont pas donné de résultats satisfaisants, et un hyménoptère local, Melipona marginata, produit un miel acidulé en faible quantité. Afin d'évaluer les possibilités de pratiquer l'apiculture en Guyane, il était nécessaire d'étudier le comportement de l'abeille africanisée dans son milieu, de déterminer les ressources de nectar et de pollen disponibles, et d'évaluer les niveaux de production de miel.

\section{Matériel et méthode}

L'étude a porté sur 54 ruches peuplées d'abeilles africanisées, de janvier 1987 à juillet 1989. Le type de ruche est le format Langstroth (très répandu en Amérique du Sud), en "grignon franc » (Ocotea rubra), bois léger et résis- tant. On a placé les ruches sur des chevrons de $50 \times 80$, eux-mêmes reposant sur des agglomérés en ciment séparés par une feuille de tôle enduite de graisse (protection contre les fourmis). Les fonds de ruches sont grillagés pour limiter l'humidité.

On a comparé trois milieux qui diffèrent par leur végétation :

- la mangrove : bande côtière en constante évolution, c'est une forêt généralement monospécifique constituée de palétuviers gris (Avicennia nitida) dans sa phase de croissance et de maturité, mais qui peut s'enrichir en palétuviers rouges (Rhisophora racemosa) dans sa phase de sénescence ;

- la savane : située entre la mangrove et la forêt dense, elle est constituée d'une strate herbacée importante, de petits arbustes et de palmiers ;

- la forêt dense ombrophile sempervirente qui couvre 95 p. 100 de la Guyane, est très hétérogène. On peut dénombrer plusieurs centaines d'espèces d'arbres différents sur une surface de $1 \mathrm{~km}^{2}$.

Pour les trois types de végétation, on a constitué deux ruchers de neufs ruches, placées dans des lieux dégagés de toute végétation herbacée, mais protégés de l'ensoleillement.

On a déterminé quelques sources de nectar et de pollen par observation des butineuses sur les fleurs à différentes heures de la journée en saisons sèches (août à novembre, mars) et humides (décembre à février, avril à juillet). Sur le plan biologique, on a observé le développement des reines, des ouvrières et des mâles d'Apis mellifera adansonii par repérage des œufs sur un cadre de couvain, ainsi que la prolificité de la reine par comptage journalier de la ponte. On a testé l'agressivité des colonies en suspendant une boule de cire devant l'entrée des ruches, et mesuré ainsi le temps que mettent les abeilles pour attaquer la boule. On a également déterminé la distance sur laquelle les abeilles attaquent une personne qui a visité une ruche.

\section{Résultats}

\section{Sources de nectar et de pollen}

Le tableau I regroupe les espèces mellifères et pollinifères observées dans les trois milieux. En général, l'ensemble des palmiers constitue une bonne source de nectar et de pollen. Certains arbres (en particulier des légumineuses genre Acacia) sont très attractifs au moment de leur floraison. On a également remarqué des butinages sur des fleurs et des feuilles d'hévéas.

\section{Comportement}

Les cycles de développement des reines, des ouvrières et des mâles sont donnés dans le tableau II. 
TABLEAU I Espèces mellifères et pollinifères observées dans les trois milieux.

\begin{tabular}{|c|c|c|c|c|c|c|}
\hline & Famille & Genre & Espèce & $\begin{array}{l}\text { Nom } \\
\text { vulgaire }\end{array}$ & Floraison & $\begin{array}{c}\text { Miel (M) } \\
\text { Pollen (P) }\end{array}$ \\
\hline Mangrove & $\begin{array}{l}\text { COMBRETACÉES } \\
\text { RHIZOPHORACÉES }\end{array}$ & $\begin{array}{l}\text { Avicennia } \\
\text { Rhizophora }\end{array}$ & $\begin{array}{l}\text { nitida } \\
\text { racemosa }\end{array}$ & palétuvier & $\begin{array}{l}\text { août } \\
\text { sept. }\end{array}$ & $\begin{array}{l}M+P \\
M+P\end{array}$ \\
\hline Savane & $\begin{array}{l}\text { ANACARDIACÉES } \\
\text { CARICACÉES } \\
\text { GRAMINÉES } \\
\text { MALVACEÉES } \\
\text { MIMOSÉES } \\
\text { MYRTACÉES } \\
\text { PALMACÉES } \\
\text { PAPILLIONACÉES }\end{array}$ & $\begin{array}{l}\text { Mangifera } \\
\text { Carica } \\
\text { Brachiaria } \\
\text { Brachiaria } \\
\text { Zea } \\
\text { Hibiscus } \\
\text { Acacia } \\
\text { Leucaena } \\
\text { Mimosa } \\
\text { Boreria } \\
\text { Eucalyptus } \\
\text { Astrocarium } \\
\text { Attalea } \\
\text { Coco } \\
\text { Mauritia } \\
\text { Oenocarpus } \\
\text { Oenocarpus } \\
\text { Arachis }\end{array}$ & $\begin{array}{l}\text { indica } \\
\text { candinamar } \\
\text { decumbens } \\
\text { humidicola } \\
\text { maize } \\
\text { esculenta } \\
\\
\text { leucocephala } \\
\text { pudica } \\
\text { verticilata } \\
\text { sp. } \\
\text { tucuma } \\
\text { regia } \\
\text { nucifera } \\
\text { flexuosa } \\
\text { bataua } \\
\text { hopii } \\
\text { hypogea }\end{array}$ & $\begin{array}{l}\text { manguier } \\
\text { papayer } \\
\text { decumbens } \\
\text { humidicola } \\
\text { maïs } \\
\text { nombo } \\
\text { acacia } \\
\text { leucaena } \\
\text { sensitive } \\
\text { tête nègre } \\
\text { eucalyptus } \\
\text { awara } \\
\text { maripa } \\
\text { cocotier } \\
\text { bâche } \\
\text { patawa } \\
\text { comou } \\
\text { arachide }\end{array}$ & $\begin{array}{l}\text { toute l'année } \\
\text { sept. } \\
\text { août } \\
\text { sept. } \\
\text { sept. } \\
\text { oct. } \\
\text { oct. } \\
\text { toute l'année } \\
\text { toute l'année } \\
\text { toute l'année } \\
\text { toute l'année } \\
\text { oct. } \\
\text { toute l'année } \\
\text { toute l'année } \\
\text { oct. } \\
\text { oct. } \\
\text { toute l'année } \\
\text { sept. }\end{array}$ & $\begin{array}{c}M \\
P \\
P \\
P \\
P \\
M+P \\
M \\
M \\
M+P \\
M+P \\
M \\
M \\
M \\
M+P \\
M+P \\
M \\
M \\
M\end{array}$ \\
\hline Forêt & $\begin{array}{l}\text { ANACARDIACÉES } \\
\text { BOMBACÉES } \\
\text { CAPRIFOLIACÉES } \\
\text { PALMACEEES }\end{array}$ & $\begin{array}{l}\text { Anacardium } \\
\text { Pachira } \\
\text { Sambucus } \\
\text { Attalea } \\
\text { Elaeis } \\
\text { Euterpe } \\
\text { Hymenaea } \\
\text { Mauritia } \\
\text { Oenocarpus } \\
\text { Oenocarpus } \\
\text { Inga } \\
\text { Pterocarpus }\end{array}$ & $\begin{array}{l}\text { giganteum } \\
\text { aquatica } \\
\text { mexicana } \\
\text { regia } \\
\text { oleifera } \\
\text { oleracera } \\
\text { courbaril } \\
\text { flexuosa } \\
\text { bataua } \\
\text { hopii } \\
\text { edulis } \\
\text { erina }\end{array}$ & $\begin{array}{l}\text { cajou } \\
\text { cacao riv. } \\
\text { sureau } \\
\text { maripa } \\
\text { palmier } \\
\text { pinot } \\
\text { courbaril } \\
\text { bâche } \\
\text { patawa } \\
\text { comou } \\
\text { pois sucré }\end{array}$ & $\begin{array}{l}\text { sept. } \\
\text { sept. } \\
\text { oct. } \\
\text { toute l'année } \\
\text { toute l'année } \\
\text { toute l'année } \\
\text { oct. } \\
\text { oct. } \\
\text { oct. } \\
\text { toute l'année } \\
\text { oct. } \\
\text { oct. }\end{array}$ & $\begin{array}{c}M \\
M \\
M \\
M \\
M+P \\
M+P \\
M \\
M+P \\
M \\
M \\
M \\
M\end{array}$ \\
\hline
\end{tabular}

Prolificité de la reine : le comptage a donné les chiffres suivants, quel que soit le milieu dans lequel les ruches sont placées : jusqu'à 4000 œufs par jour en saison sèche. La reine réduit ensuite sa ponte en saison humide : environ 1500 œufs par jour.

TABLEAU II Les cycles de développement des reines, ouvrières et mâles (en jours).

\begin{tabular}{|l|c|c|c|}
\hline & Reines & Ouvrières & Mâles \\
\hline CEuf & 3 & 3 & 3 \\
$\begin{array}{l}\text { Larve : alvéole } \\
\text { ouvert }\end{array}$ & 5 & 5 & 6 \\
$\begin{array}{l}\text { Larve : alvéole } \\
\text { operculé } \\
\begin{array}{c}\text { Emergence de } \\
\text { l'insecte }\end{array}\end{array}$ & 15 & 18 & 15 \\
\hline
\end{tabular}

Heures de butinage : l'activité est importante dès les premières lueurs du jour, augmente juqu'à $9 \mathrm{~h}$, diminue pendant les heures chaudes de la journée, puis reprend vers $16 \mathrm{~h}$. On a remarqué que certaines plantes n'étaient visitées que très tôt le matin, notamment Mimosa pudica et les palmiers.

Butinage sous la pluie: lorsque la pluie n'est pas trop forte, l'activité des abeilles est peu réduite. La température diurne ne descend jamais en dessous de $21^{\circ} \mathrm{C}$.

Agressivité : le test de la boule de cire a montré un temps de réaction de 5 secondes, identique selon les colonies. En revanche, l'intensité de l'attaque varie beaucoup d'une colonie à l'autre, à population sensiblement égale. La distance de poursuite a varié de 500 à $1000 \mathrm{~m}$.

Essaimage : Apis mellifera adansonil est naturellement porté à l'essaimage. Dans les trois milieux observés, on a estimé qu'environ 30 p. 100 des ruches essaiment chaque année. La désertion, abandon pur et simple de la 
ruche en présence de miel et de couvain, est assez fréquente. On donne, pour l'année 1988, le nombre de ruches ayant essaimé ou ayant été abandonnées :

- essaimage : mangrove $=6$, savane $=4$, forêt $=7$;

- désertion : mangrove $=4$, savane $=1$, forêt $=2$.

\section{Production}

Même si les sources de pollen et de nectar sont disponibles tout au long de l'année, elles sont bien plus abondantes en saison sèche. En dehors de cette période, les heures de butinage sont réduites du fait de la pluviosité, le nectar est dilué et suffit parfois à peine à la consommation des colonies. Les niveaux de production sont présentés dans le tableau III.

TABLEAU III Niveaux de production.

\begin{tabular}{|c|c|c|c|c|c|}
\hline & \multirow{2}{*}{$\mathrm{Nb}$. ruches } & \multicolumn{3}{|c|}{ Quantité de miel (en kg) } & \multirow{2}{*}{$\begin{array}{l}\text { Moy./ruche } \\
1987 \text { et } 1988 \\
\quad(e n k g)\end{array}$} \\
\hline & & sept. & déc. & mars & \\
\hline Mangrove & 18 & 234 & 0 & 0 & 13 \\
\hline Savane & 18 & 300 & 356 & 64 & 40 \\
\hline Forêt & 18 & 266 & 306 & 76 & 36 \\
\hline
\end{tabular}

Les colonies les plus populeuses ont produit jusqu'à $60 \mathrm{~kg}$. Les ruches placées en savane ont produit deux qualités de miel : l'un clair (septembre), l'autre ambré (octobre-novembre). Le miel de mangrove est ambré, celui produit en forêt est très foncé quelle que soit l'époque de récolte.

\section{Discussion}

\section{Sources de nectar et de pollen}

La période de floraison la plus favorable à l'apiculture s'étend d'août à décembre. En mangrove, les palétuviers ne fleurissent que deux mois, et les colonies sont privées de sources de nectar et de pollen pendant le reste de l'année. En savane, la diversité des sources mellifères et pollinifères permet le butinage tout l'année. En forêt, SABATIER (7) a constaté qu'environ 80 p. 100 des espèces fleurissent en saison sèche. Cependant, la présence importante des palmiers offre une source de nectar et de pollen tout le reste de l'année.

La liste proposée n'est nullement exhaustive. Les espèces visitées sont certainement très nombreuses et mériteraient une étude plus approfondie en Guyane, d'autant plus que d'autres études ont été menées sur les sources de nectar en milieu tropical (2). De plus, l'apiculture en Guyane, contrairement au Brésil, est sédentaire. II n'existe pas de culture industrielle permettant des transhumances pour suivre les floraisons et accroître ainsi la production.

\section{Cycles de développement}

Les cycles de développement des reines et des ouvrières sont plus rapides que chez les abeilles européennes. La différence se situe dans la réduction du stade larvaire, due probablement aux conditions climatiques (température élevée). En ce qui concerne les mâles, le temps de développement est identique à celui des races européennes. Les chiffres obtenus se rapprochent des expérimentations menées au Vénézuéla (5) et en Afrique du Sud (11).

\section{Prolificité de la reine}

Les résultats confirment la remarquable prolificité des reines africanisées (9). Chez une reine italienne (Apis mellifera ligustica), la quantité d'œufs pondus par jour, en condition de bonne miellée, n'excède pas 2000.

\section{Butinage d'Apis mellifera andansonii}

II est très efficace. Elle butine très tôt le matin et tard le soir, en toute saison sauf si la pluie est forte. Cette capacité de butiner à des intensités lumineuses très faibles constitue une parfaite adaptation aux heures de sécrétion du nectar des plantes visitées. SMITH (8) a observé des sécrétions de nectar pendant les heures les plus fraîches de la journée en Afrique, et même la nuit pour certaines espèces.

\section{Réactions de défense}

Les abeilles africanisées réagissent plus vite et en plus grand nombre à une attaque extérieure que des abeilles européennes (1): le test de la boule de cire donne un temps de réaction de $20 \mathrm{~s}$, et la distance de poursuite ne dépasse pas $50 \mathrm{~m}$ chez Apis mellifera ligustica. Les réactions de défense sont aussi rapides qu'imprévisibles, et on n'a pas pu déterminer quel facteur précis pouvait influencer leur comportement (mis à part l'importance de la colonie et la quantité de' miel présent dans la ruche; presque toujours, plus la ruche est populeuse et plus il y a du miel, plus les abeilles sont agressives). Cette agressivité est renforcée par la chaleur et il est préférable de manipuler les ruches tôt le matin ou en soirée.

\section{Essaimages et abandons}

Les essaimages et abandons des colonies d'abeilles africanisées confirment l'importance de ce phénomène $(3$, 13). La température élevée et une floraison continue rendent possible, du moins en forêt et en savane, la subsistance de colonies faibles.

L'essaimage et l'abandon fréquent, la forte prolificité des reines et la sécrétion continuelle de nectar ont certainement contribué à l'extension rapide de ces abeilles en Amérique du Sud.

\section{Production}

Les niveaux de production atteints sont très satisfaisants et rejoignent ceux obtenus au Brésil (12). Cependant, il paraît évident que la mangrove ne constitue pas un lieu intéressant pour pratiquer une apiculture sédentaire ; les rendements sont faibles et l'essaimage fréquent. Des niveaux de production plus élevés ont été atteints en mangrove au Surinam (4), et des lieux plus productifs 
pourraient être prospectés le long du littoral guyanais. II serait ainsi possible de transhumer en période de floraison, le miel étant différent.

La savane, d'accès facile et riche en composition floristique, est le milieu naturel le plus favorable à l'apiculture. La forêt profonde n'est accessible que par voie d'eau ou par les quelques pénétrantes forestières existantes, il semble donc difficile d'y pratiquer une apiculture rentable.

L'amélioration de la production pourrait être obtenue par la sélection de souches productives mais moins agressives (élevage de reines à partir de ces souches).

\section{Conclusion}

L'apiculture en Guyane française peut représenter une voie de diversification intéressante pour quelques exploitants agricoles. Avec 50 ruches correctement gérées en apiculture sédentaire en savane, il est possible de produire deux tonnes de miel vendues au détail à $50 \mathrm{~F} / \mathrm{kg}$.

Le marché local est cependant restreint : $11 \mathrm{t}$ ont été importées en 1988 et représentent la totalité de la consommation guyanaise. Mais le miel est un aliment très facile à stocker et à transporter, et celui de Guyane pourrait venir enrichir la gamme des miels exotiques proposés sur les marchés européens.

CANOVA (M.), GAUCHER (B.). Note on beekeeping and africanized honeybees in French Guyana. Revue Élev. Méd. vét. Pays trop., 1991, 44 (4) : 497-500

Beekeeping hardly exists in French Guyana. In order to make clear the possibilities of developping this activity we have made a survey, comparing the behaviour of the africanized honeybee, the various sources of pollen and nectar, and the levels of production, in three natural surroundings quite different from one another : the mangrove swamps, the savanna and the forest. Key words: Honeybee - Behaviour - Feeding - Pollen - Nectar - French Guyana,

\section{Bibliographie}

1. COLLINS (A.M.), RINDERER (T.E.), BOLTEN (A.B.). Colony defense by africanized and european honey bees. Science, 1982, $218: 72-74$.

2. CRANE (E.). Honey sources of some tropical and subtropical countries. Bee world, 1973, 54 : 117-186.

3. FLETCHER (D.J.C.), TRIBE (G.D.). Swarming potential of the African bee Apis mellifera adansonii. These proceedings, 1977. P. 25-34.

4. GARD (W.), TAYLOR (O.R.). Beekeeping in the Guianas. Bee Resarch Association, 1979.

5. HARBO (J.R.), BOLTEN (A.B.), RINDERER (.E.), COLLINS (A.M.). Development periods for eggs of africanized and european honey bees 1981

6. MICHENER (C.D.). The brazilien bee problem. Ann. Rev. entomol., $1975,20: 399-416$

7. SABATIER (D.). Saisonnalité et déterminisme du pic de fructification en forêt guyannaise. Revue Écol. (Terre et vie), 1985, 40:289-320.

8. SMTTH (T.). Beekeeping observation in Tanganyka 1949-1957. Bee world, 1958, 39:29-36.

9. STEINHOBEL (F.). Swarming on the aloes and its utilization for making increase. These proceedings, 1977. P. 152-156.

10. TAYLOR (OR), WILLIAMSON (B ) Current status of the africanized honey bee in Northern South America. Am. Bee J., 1975, 115 : 92-93 ; 98-99. 11. TRIBE (G.D.), FLETCHER (D.J.C.). Rate of development of the workers for Apis mellifera andansonii. Apimondia International symposium. 1976. P. 115-119.

12. WIESE (H.). Nova apicultura. Porto Alegre, Livraria e editora Agropecuaria Ltda, 1985. P. 459-461.

13. WINSTON (M.L.). Swarming, after swarming and reproductive rate of unmanaged honeybee colonies (Apis mellifera). Insectes sociaux, 1980, 27 $391 ; 398$.

\section{La place de la brebis dans le circuit de la viande à Maroua (extrême nord du Cameroun)}

\author{
E. Thys ${ }^{1}$
}

THYS (E.). La place de la brebis dans le circuit de la viande à Maroua (extrême-nord du Cameroun). Revue Élev. Méd. vét. Pays trop., 1991, 44 (4) : 500-502

L'importance de la brebis dans le circuit de la viande dans la ville de Maroua (extrême-nord du Cameroun) est mise en évidence à l'aide d'un échantillon prélevé à l'abattoir. 89,6 p. 100 des ovins abattus sont des

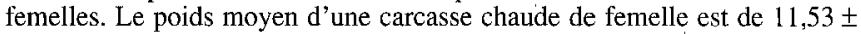
$2,874 \mathrm{~kg}$ et l'indice de compacité est de $0,21 \pm 0,040$. Une très forte homogénéité de poids et de gabarit existe entre les classes d'âge. On en déduit que les carcasses de 11 à $12 \mathrm{~kg}$ produites économiquement répondraient mieux aux besoins du marché de la viande. Les reproductrices à réformer pourraient recevoir une alimentation de finition bon marché. Mots clés : Ovin - Brebis - Viande - Étude de marché - Carcasse - Rendement à l'abattage - Cameroun.

La plupart des études effectuées sur l'embouche de petits ruminants en Afrique portent sur les performances des mâles castrés ou non.

Une enquête menée en 1985 dans la ville de Maroua (extrême-nord du Cameroun) a montré que, si les béliers avaient une très grande importance dans le contexte de l'abattage familial, principalement lors de manifestations à caractère sacrificiel (Tabaski...) les brebis étaient nombreuses à être abattues pour le circuit de la viande, que ce soit à l'abattoir ou parmi les bouchers de quartier (3) (tabl. I).

Des sondages effectués en 1988 sur les marchés du département du Diamaré (4) permettent d'analyser la répartition fine par classes d'âge et de sexe des ovins mis en vente (fig. 1).

TABLEAU I Répartition par sexe des abattages de moutons dans les concessions, à l'abattoir et chez les bouchers de quartier (d'après Thys et Ekembé, 1988).

\begin{tabular}{|l|rr|rr|rr|}
\hline \multicolumn{1}{|c|}{ Sexe } & \multicolumn{2}{|c|}{$\begin{array}{c}\text { Abattages } \\
\text { familiaux }\end{array}$} & \multicolumn{2}{|c|}{$\begin{array}{c}\text { Abattages } \\
\text { à l'abattoir }\end{array}$} & $\begin{array}{c}\text { Abattages } \\
\text { bouchers de } \\
\text { quartier }\end{array}$ \\
\cline { 2 - 7 } & $\mathrm{N}$ & p. 100 & $\mathrm{~N}$ & p. 100 & $\mathrm{~N}$ & p. 100 \\
\hline Mâles & 1010 & 94,5 & 44 & 26,6 & 230 & 41,1 \\
\hline Femelles & 59 & 5,5 & 121 & 73,4 & 330 & 58,9 \\
\hline Total & 1069 & 100,0 & 165 & 100,0 & 560 & 100,0 \\
\hline
\end{tabular}

1. Institut de médecine tropicale Prince Léopold, Service de production animale tropicale, Nationalestraat, 155, 2000-Antwerpen, Belgique.

Reçu le 14.1.1991, accepté le 10.12.1991. 